\title{
The nature of super-metal-rich stars ${ }^{\star}$
}

\section{Detailed abundance analysis of 8 super-metal-rich star candidates}

\author{
S. Feltzing ${ }^{1}$ and G. Gonzalez ${ }^{2}$ \\ 1 Lund Observatory, Box 43, 22100 Lund, Sweden \\ 2 Astronomy Department, University of Washington, PO Box 351580, Seattle, WA 98195, USA
}

Received 24 May 2000 / Accepted 17 November 2000

\begin{abstract}
We provide detailed abundance analyses of 8 candidate super-metal-rich stars. Five of them are confirmed to have $[\mathrm{Fe} / \mathrm{H}]>0.2 \mathrm{dex}$, the generally-accepted limit for super-metal-richness. Furthermore, we derive abundances of several elements and find that the stars follow trends seen in previous studies of metal-rich stars. Ages are estimated from isochrones and velocities calculated. We find that there do exist very metal-rich stars that are older than 10 Gyr. This is contrary to what is found in several recent studies of the galactic age-metallicity relation. This is tentative evidence that there might not exist a one-to-one relation between age and metallicity for all stars. This is not surprising considering the current models of the independent evolution of the different galactic components. We also find that one star, HD 182572, could with $\sim 75 \%$ chance be a thick disk star with, for the thick disk, an extremely high metallicity at 0.34 dex. This star is, intriguingly, also somewhat enhanced in the $\alpha$-elements.
\end{abstract}

Key words. stars: abundances, fundamental parameters, late-type, individual HD 10780, HD 32147, HD 99491, HD 104304, HD 121370, HD 145675, HD 196755, HD 182572 - Galaxy: solar neighbourhood

\section{Introduction}

The very metal-rich dwarf stars in the solar neighbourhood have historically not attracted as much attention as the more metal-poor (solar like and halo stars) stars which tell us about the early phases of the chemical evolution of our galaxy. The properties of metal-rich stars are important when we try to interpret integrated spectra from metal-rich stellar populations, such as the Bulge and giant elliptical galaxies. A small group of so called super-metalrich (SMR) stars have played a significant role in shaping the conceptions of such populations. Famous examples are the dwarf HD 32147 (HR 1614) and the giant $\mu$ Leonis. In the review by Taylor (1996) - the latest paper in a long series started in the 1960s - SMR stars are discussed in great detail, in particular, the reality of extremely high $[\mathrm{Fe} / \mathrm{H}]$. Taylor found that no giant star fulfills the criteria for SMR-ness that he sets and only a handful of dwarf stars do, and that most of them are candidates rather than firm members in this class. $\mu$ Leonis has, however, been studied by several groups using high-resolution spectroscopy, a recent example being Smith \& Ruck (2000), who find that

Send offprint requests to: S. Feltzing,

e-mail: sofia@astro.lu.se

* Based on observations obtained at the McDonald Observatory. the star is indeed super-metal-rich with $[\mathrm{Fe} / \mathrm{H}]=+0.29$. Thus, the question of the reality of super-metal-rich giants is still very much alive and each case has to be judged on its own.

The exact definition of super-metallicity has, as reviewed by Taylor (1996), varied. Spinrad \& Taylor (1969) adopted +0.2 dex as the lower limit, based on the overall metallicity of the Hyades, which they found to be +0.2 dex. The metallicity for the Hyades has recently been revised (Taylor 1994; Cayrel de Strobel 1997) to +0.1 dex. Even values as low as 0.0 dex have been quoted. This has resulted in classes of stars that sometimes are regarded as SMR and sometimes not. Taylor rectified this unsatisfactory situation by adopting the original +0.2 dex as the threshold on the grounds that no giant stars had been shown to have a metallicity higher than this value (but see Castro et al. 1997; Smith \& Ruck 2000). Taylor (1996) defines a star to be SMR if it has $[\mathrm{Fe} / \mathrm{H}]>0.20$ with $95 \%$ confidence. He also adopts $[\mathrm{Fe} / \mathrm{H}]$, i.e. the iron abundance, as the measure of "metallicity" rather than the more general $[\mathrm{Me} / \mathrm{H}]$. As an aside one may note that a second terminology is also in use - Very Strong-Lined (VSL) star. This term implies just that the star has strong lines and might therefore be a SMR candidate. This is a particularly useful term when working with low resolution spectra. 
SMR stars have attracted more attention recently due to their possible connection with extra-solar planets, e.g. Gonzalez (2000 and references cited therein), Fuhrmann et al. (1997, 1998). Gonzalez (2000) has shown that the solar-type parent stars of extra-solar planets are more metal-rich on average compared to the general field star population. In particular, the very short period systems are either above the SMR limit or near it. By comparing them to the SMR stars we may gain insight as to the relationship between planets in short-period orbits and the SMR-ness of the parent star.

A few other recent studies have targeted known SMR candidates and stars with high $[\mathrm{Me} / \mathrm{H}]$ (as derived from photometry): Feltzing \& Gustafsson (1998); Castro et al. (1997); and McWilliam \& Rich (1994). In general the abundance ratios seem to continue the trends of the disk population. However, no detailed theoretical predictions for Galactic chemical evolution exists for $[\mathrm{Fe} / \mathrm{H}]>0.2 \mathrm{dex}$, so the interpretation of the observed abundance trends for metal-rich stars is still pending.

The combination of abundance ratios with kinematical data may give us additional clues. For example, we can study stars on highly eccentric orbits which trace the evolution in the Galactic disk closer to the Galactic centre. Not much is known about these stars, but there are some very intriguing observations: Barbuy \& Grenon (1990) found that dwarf stars on very eccentric orbits contained much more oxygen than what was expected from standard models of Galactic chemical evolution of the disk, and Edvardsson et al. (1993) found large spreads and "upturns" for certain elements, $\mathrm{Na}, \mathrm{Si}, \mathrm{Ti}, \mathrm{Al}$, for stars with 0.0 dex $<[\mathrm{Fe} / \mathrm{H}]<0.2$ dex. The trends for $\mathrm{Na}$, Si and $\mathrm{Ti}$ were confirmed up to $\sim 0.4$ dex by Feltzing \& Gustafsson (1998). They concluded that the "upturn" in Na abundances relative to $\mathrm{Fe}$ is not due to a mixture of stars born at different distances from the Galactic centre.

In this paper we investigate, by means of detailed spectroscopic analyses, the metallicities as well as the abundance of several elements for 8 dwarf stars selected from the meticulous review of SMR candidates by Taylor (1996).

The paper is organized as follows: in Sects. 2 and 3 we detail the observations and the selection of program stars, as well as reductions and measurements; Sect. 4 discusses the detailed abundance analysis, Sect. 5 presents the abundances element by element, in Sect. 6 we derive ages for the stars and discuss the age-metallicity relation in the solar neighbourhood, Sect. 7 discusses the kinematics of the stars in our sample and which galactic component they belong to, Sect. 8 provides a short discussion of the SMR-planet connection and, finally, Sect. 9 summarizes our findings.

\section{Selection of program stars and observations}

Stars observable from the Northern Hemisphere were selected from Taylor's 1996 list of candidate SMR dwarf and subgiant stars. Spectra were obtained with the Sandiford

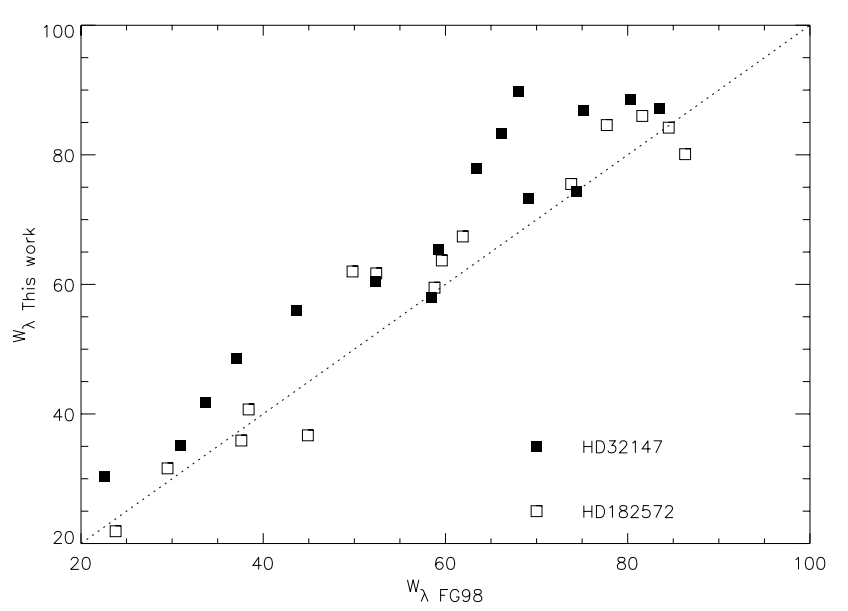

Fig. 1. Comparison of measurements of $W_{\lambda}(\AA)$ in this work and in Feltzing \& Gustafsson (1998) for HD 32147 and HD 182572

cassegrain echelle spectrograph (McCarthy et al. 1993) attached to the $2.1 \mathrm{~m}$ Struve telescope at McDonald Observatory during three runs: 1996 June, 1996 August and 1996 December. Exposure times were typically $5 \mathrm{~min}$ each, resulting in signal-to-noise $(S / N)$ ratios per pixel averaging near 250. A spectrum of a Th-Ar lamp was obtained following each star spectrum, ensuring accurate wavelength calibration. The resolving power (measured on the Th-Ar emission line spectra) averages near $R=45000$.

\section{Reductions and measurements}

The spectra were reduced with the standard software available within the CCDRED and ECHELLE packages of NOAO IRAF ${ }^{2}$. The steps included bias subtraction, flat fielding, extraction of one-dimensional spectra, wavelength calibration, and continuum normalization. Additional details concerning the quality of the data resulting from the Sandiford spectrograph can be found in Gonzalez \& Lambert (1996) and Gonzalez (1998).

Equivalent widths $\left(W_{\lambda}\right)$ were measured using the SPLOT task in IRAF. The lines were measured both by simply integrating the line and also by fitting a Gaussian to the line profile. Most lines were measured twice and some up to four times due to overlap of the spectral orders. As the final adopted value of $W_{\lambda}$ we used the mean of the measurements. In these cases the measurement errors are typically no more than a few percent.

In Fig. 1 we compare the measured values of $W_{\lambda}$ for HD 32147 and HD 182572 with those measured by Feltzing \& Gustafsson (1998). For HD 182572 the agreement is good, while for HD 32147, our coolest star, we measure significantly larger $W_{\lambda}$. This difference is most likely due to the lower resolution used in this work. See also the two

\footnotetext{
${ }^{2}$ IRAF is distributed by National Optical Astronomy Observatories, operated by the Association of Universities for Research in Astronomy, Inc., under contract with the National Science Foundation, USA.
} 
examples of stellar spectra shown in Fig. 3 from which it is clear that HD 32147, but also to some extent HD 145675, shows a much richer spectrum than the other stars. Since these stars are cool, there will naturally be more molecular lines and low-excitation atomic lines that will cause blending problems.

\section{Analysis}

We have performed a standard Local Thermodynamic Equilibrium (LTE) analysis, strictly differential with respect to the Sun, to derive chemical abundances from the measured values of $W_{\lambda}$. Spectrum synthesis was not employed in the present study.

\subsection{Model atmospheres}

To generate the model atmospheres we used the MARCS program, first described by Gustafsson et al. (1975). The program has been further developed and updated in order to handle the line blanketing of millions of absorption lines more accurately (Asplund et al. 1997). The following assumptions enter into the calculation of the models: the atmosphere is assumed to be plane-parallel and in hydrostatic equilibrium, the total flux (including mixing-length convection) is constant, the source function is described by the Planck function at the local temperature with a scattering term, the populations of different excitation levels and ionization stages are governed by LTE.

Since our analysis is strictly differential relative to the Sun, we have used a solar model atmosphere calculated with the same program as the stellar models - this in order to keep the analysis truly differential and thus in spite of the fact that the empirically derived HolwegerMüller model better reproduces the solar observed limb darkening (Blackwell et al. 1995).

\subsection{Line data}

Since we did not have observations of the solar spectrum for all of the lines available in the stellar spectra, we measured solar line strengths in the Kurucz et al. (1984) Solar Flux Atlas. The spectrum from the Flux Atlas was first degraded by binnning and then convolved with a Gaussian profile to match the instrumental profile. To decide on the exact values of the convolution, we used three portions of a spectrum of reflected sunlight from Vesta. The Flux Atlas spectrum was convolved and then compared with the Vesta spectrum. The goodness of the fit was decided upon by inspection. The values of $W_{\lambda}$ for all our lines were measured in the degraded spectrum. They were then used to determine the astrophysical $\log g f$ values, Table A1.

We consider different line broadening mechanisms in our calculations; van der Waals damping, radiation damping, thermal Doppler broadening and broadening by microturbulence. The van der Waals broadening is calculated with the classical Unsöld formula. Enhancement factors to this value were compiled from the literature and are given
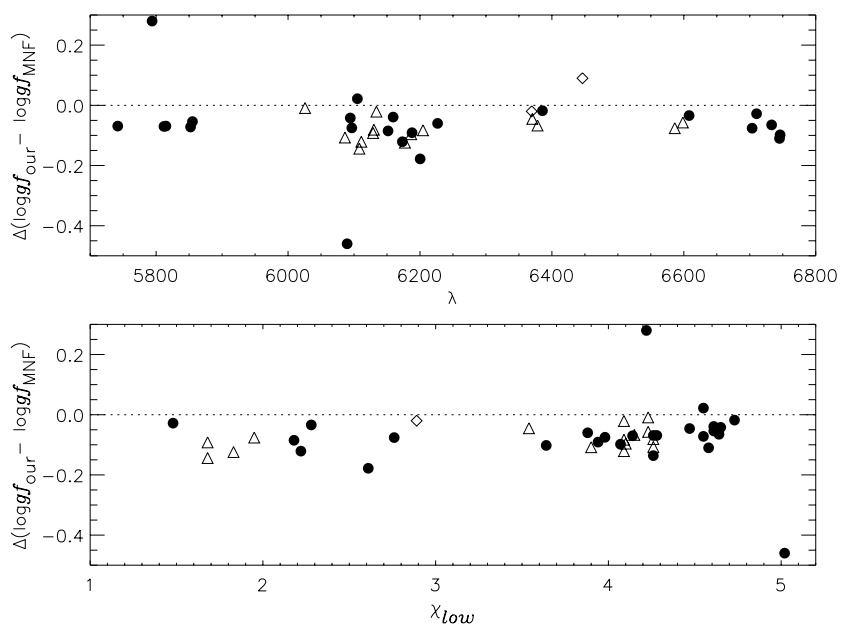

Fig. 2. Comparison of $\log g f$-values as derived in this study and in Neuforge-Verheecke \& Magain (1997) study of $\alpha$ Cen $\mathrm{A}$ and B. Fe I lines are denoted by $\bullet$, Fe II by $\diamond$ and Ni I lines by $\triangle$

in Table A1. For Fe we use values from Hannaford et al. (1992) and Holweger et al. (1991), for Ca, and for V from Neuforge (1992). For the remaining lines we use a value of 2.5, according to Mäckle et al. (1975). The values used for the enhancement factor do not, in general, influence the results, e.g. a change from 2.5 to 1.4 does not alter most abundances by more than 0.01 dex.

We have also compared our $\log g f$-values derived from the solar spectrum with those derived in a similar way, but using a Holweger-Müller solar model, by NeuforgeVerheecke \& Magain (1997), Fig. 2. Our $\log g f$-values are 0.07 dex lower for Fe I lines and 0.04 dex lower for the Ni I lines than those derived by Neuforge-Verheecke \& Magain (1997). Considering the different approaches to the derivation of the astrophysical $\log g f$-values we consider the agreement good. It is also reassuring that no trends are found either with wavelength or excitation potential, see Fig. 2.

\subsubsection{Selection of lines}

Selecting stellar lines which are free from blends is crucial for deriving accurate elemental abundances. To account for telluric lines we simply over-plotted each stellar spectrum with a spectrum of a hot star observed during the same night as the stellar spectrum was taken and discarded lines that were contaminated. To avoid blends from unidentified photospheric lines the solar spectrum was carefully inspected and the line-list by Moore consulted.

Care in the selection of lines is also of importance for the determination of surface gravities by means of ionization equilibrium (i.e., abundances derived from $\mathrm{Fe}$ I and Fe II lines give the same iron abundance). We have inspected the shape of the Fe II lines in all the stars when a line is observed in more than two stars. This inspection led us to discard the lines at 6386.72 and $7449.33 \AA$, Fig. 3 . 

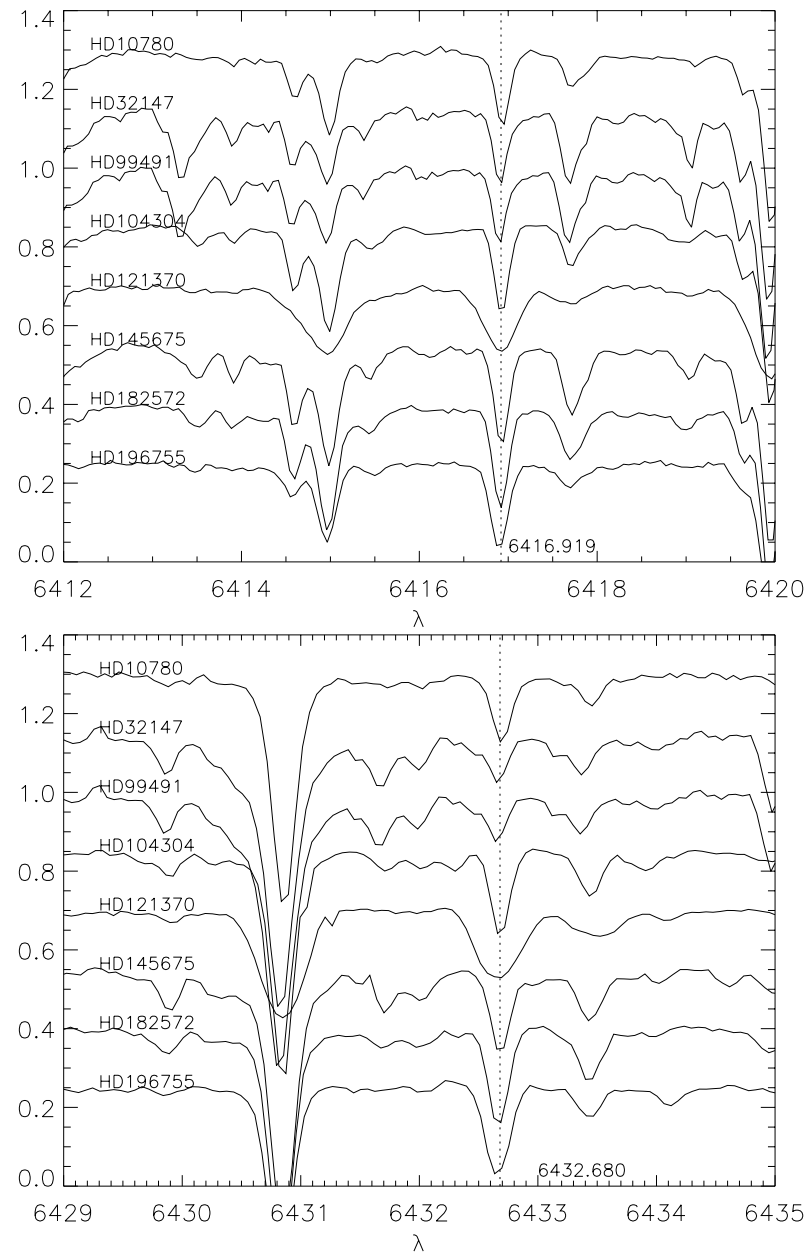

Fig. 3. Two portions of the stellar spectra, showing the regions around the two Fe II lines at 6416.91 and $6432.68 \AA$. The spectra have been arbitrarily displaced in intensity and also along the $x$-axis to the laboratory wavelengths. The positions of the Fe II lines are marked with dotted lines. Note the different scales on the $x$-axes

A line at $5823.15 \AA$ was also discarded, although only measured in two stars, since it gave anomalously high iron abundances and clearly suffered from blends. The line at $6416.91 \AA$ gave rather high iron abundances in HD 10780 , HD 32147 and HD 145675. From our spectra we could not, however, conclude that this line is compromised by a blend (see Fig. 3) and it was therefore kept in the analysis, but only in those stars where it did not diverge significantly. Our final selection of lines, as well as the parameters used in the abundance analysis, are given in Table A1.

\subsection{Fundamental parameters of model atmospheres}

In order to construct the stellar model atmospheres we need the effective temperature, surface gravity, metallicity and microturbulence for each star. These were all derived from the stellar spectra themselves.

Effective temperature Initial estimates of effective temperatures for the stars were determined using the photometric calibrations by Alonso et al. (1996). These estimates were iterated until excitation energy equilibrium was achieved. The plots from which the final temperatures were derived are shown in Fig. 4, and the final adopted temperatures are given in Table 1.

Surface gravity Surface gravities were determined by requiring ionization equilibrium for $\mathrm{Fe}$ abundances derived from Fe I and Fe II lines. We adjusted $\log g$ until the iron abundance derived from Fe I and Fe II lines gave the same Fe abundance, compare Fig. 4.

Metallicity Our first estimates were taken from Taylor (1996). The $[\mathrm{Fe} / \mathrm{H}]$ were iterated until the metallicity used in constructing the atmosphere and the derived $[\mathrm{Fe} / \mathrm{H}]$ agreed.

Microturbulence The microturbulence parameter, $\xi_{\mathrm{t}}$, which is introduced to account for unknown line broadening mechanisms, affects strong and weak absorption lines differently. For weak lines only the shape, and not the $W_{\lambda}$ is affected, but for strong lines the line strength increases when $\xi_{\mathrm{t}}$ is increased. We use these trends to constrain the value of $\xi_{\mathrm{t}}$.

We started with $\xi_{\mathrm{t}}=1.00 \mathrm{~km} \mathrm{~s}^{-1}$ and, after inspecting plots of $[\mathrm{Fe} / \mathrm{H}]$ and $[\mathrm{Ni} / \mathrm{H}]$ as a functions of $W_{\lambda} / \lambda$ (reduced equivalent width), varied the value of $\xi_{\mathrm{t}}$ until all lines, weak and strong, yielded the same abundance. The final values used in the abundance analysis are given in Table 1.

\subsection{Comparison/verification with calibrations of uvby $-\beta$ photometry}

As a further check of our final stellar parameters we have derived $T_{\text {eff }}, \log g$, and [Fe/H] from the self-consistent calibration of $T_{\text {eff }}, \log g$ and $[\mathrm{Fe} / \mathrm{H}]$ by Olsen (1984), Table 2. The agreement is in general good.

\subsection{Comparison with other studies}

The stars in our study have been included in few, if any, abundance studies. However, HD 32147 and HD 182572 have been extensively studied. HD 32147 has been especially difficult to analyze, because it is a cool $\mathrm{K}$ dwarf star with strong lines. This is amply exemplified by the comparison of our $W_{\lambda}$ measurements and abundances with those of Feltzing \& Gustafsson (1998). In Table 3 we compare our results to theirs. As expected (from the comparison of $W_{\lambda}$ ) the abundances for HD 32147 are larger in our study than in theirs. In this work we impose ionization equilibrium in order to derive the surface gravity of the star. This affects in particular the abundances derived from HD 32147, but also some of the species, i.e. Fe, Co and $\mathrm{Ni}$, for HD 182572. For a discussion of stellar abundances in K dwarf stars, that for HD 32147 supersedes the current analysis, we refer the reader to Thorén \& Feltzing (2000). 
Table 1. Stellar parameters for the program stars. Magnitudes, colours and parallaxes are from the Hipparcos catalogue (ESA 1997). The spectral types are from the SIMBAD database. Effective temperatures, surface gravities, $[\mathrm{Fe} / \mathrm{H}]$ and microturbulence parameters as determined in this study

\begin{tabular}{llllllllllll}
\hline & & Sp.T. & $V$ & $B-V$ & $\pi$ & $\sigma_{\pi}\left(\sigma_{\pi} / \pi\right)$ & $T_{\text {eff }}$ & $\log g$ & {$[\mathrm{Fe} / \mathrm{H}]$} & $\begin{array}{c}\xi_{\mathrm{t}} \\
\mathrm{km} \mathrm{s}^{-1}\end{array}$ \\
\hline HD 10780 & HR 511 & K0V & 5.63 & 0.804 & 100.24 & $0.68(0.007)$ & 5300 & 4.13 & -0.02 & 1.00 \\
HD 32147 & HR 1614 & K3V & 6.22 & 1.049 & 113.46 & $0.82(0.007)$ & 4680 & 4.00 & 0.28 & 0.50 \\
HD 99491 & HR 4414A & K0IV & 6.49 & 0.778 & 56.59 & $1.40(0.025)$ & 5300 & 4.12 & 0.20 & 1.00 \\
HD 104304 & HR 4587 & G9IV & 5.54 & 0.760 & 77.48 & $0.80(0.01)$ & 5400 & 4.12 & 0.16 & 1.15 \\
HD 121370 & HR 5235 & G0IV & 2.68 & 0.580 & 88.71 & $0.75(0.008)$ & 6000 & 3.66 & 0.25 & 2.00 \\
HD 145675 & & K0V & 6.61 & 0.877 & 55.11 & $0.59(0.011)$ & 5300 & 4.50 & 0.47 & 1.00 \\
HD 182572 & HR 7373 & G8IV & 5.17 & 0.761 & 66.01 & $0.77(0.012)$ & 5400 & 4.00 & 0.35 & 1.10 \\
HD 196755 & HR 7896 & G5IV+ & 5.07 & 0.702 & 33.27 & $0.82(0.02)$ & 5700 & 4.00 & 0.02 & 1.50 \\
\hline$\alpha$ Cen A ${ }^{1}$ & & & & & & & 5830 & 4.34 & 0.24 & 1.09 \\
$\alpha$ Cen B & & & & & & & 5225 & 4.51 & 0.23 & 1.00 \\
\hline
\end{tabular}

1. $T_{\text {eff }}, \log g$ and $\xi_{\mathrm{t}}$ from Neuforge-Verheeke \& Magain (1997).

Table 2. Strömgren photometry and stellar parameters derived from the photometry thorough the calibration by Olsen (1984). References; O93 = Olsen (1993), O94a = Olsen (1994a), O94b = Olsen (1994b), GO = Gronbech \& Olsen(1997)

\begin{tabular}{lccclcc}
\hline ID & $b-y$ & $m_{1}$ & $c_{1}$ & ref. & \multicolumn{2}{c}{ Olsen $(1984)$} \\
& & & & & $T_{\text {eff }}$ & $\log g$ \\
\hline HD 10780 & 0.468 & 0.316 & 0.327 & O93 & 5431 & 4.27 \\
HD 32147 & 0.601 & 0.634 & 0.236 & O94a & 4614 & 4.57 \\
HD 99491 & 0.484 & 0.335 & 0.362 & O93 & 5347 & 4.12 \\
HD 104304 & 0.469 & 0.313 & 0.345 & O94a & 5437 & 4.19 \\
HD 121370 & 0.370 & 0.202 & 0.533 & GO & 6205 & 3.92 \\
HD 145675 & 0.537 & 0.336 & 0.438 & O93 & 4852 & 4.55 \\
HD 182572 & 0.465 & 0.299 & 0.381 & O93 & 5495 & 4.07 \\
HD 196755 & 0.432 & 0.220 & 0.381 & O94b & 5642 & 3.98 \\
\hline
\end{tabular}

Table 3. Comparison of results from this study with those of Feltzing \& Gustafsson (1998), FG98, for HD 32147 and HD 182572

\begin{tabular}{lrrrr}
\hline & \multicolumn{2}{c}{ HD 32147 } & \multicolumn{2}{c}{ HD 182572 } \\
& This work & FG98 & This work & FG98 \\
\hline Al I & 0.48 & 0.25 & 0.55 & 0.53 \\
Si I & 0.36 & 0.48 & 0.49 & 0.51 \\
Ca I & & 0.01 & & 0.42 \\
Sc II & - & 0.49 & 0.36 & 0.36 \\
Ti I & 0.66 & 0.11 & 0.32 & 0.50 \\
V I & 0.95 & -0.18 & & \\
Cr I & 0.50 & 0.10 & 0.40 & 0.43 \\
Fe I & 0.28 & 0.22 & 0.34 & 0.42 \\
Fe II & 0.24 & 0.61 & 0.32 & 0.08 \\
Co I & 0.56 & 0.39 & 0.47 & 0.58 \\
Ni I & 0.29 & 0.57 & 0.36 & 0.46 \\
\hline
\end{tabular}

Table 4. Comparison for HD 121370 of results from this study with those of Edvardsson et al. (1993). The second line for Fe II give the iron abundance derived if the line at $6416.91 \AA$ is excluded

\begin{tabular}{lrr}
\hline & This work & Edv.93 \\
\hline Na I & 0.50 & 0.45 \\
Si I & 0.40 & 0.31 \\
Ca I & & 0.23 \\
Ti I & 0.22 & 0.32 \\
Fe I & 0.24 & 0.19 \\
Fe II & 0.19 & 0.25 \\
& 0.22 & \\
Ni I & 0.31 & 0.30 \\
\hline
\end{tabular}

Gonzalez et al. (1999) found HD 145675 (14 Her) to have $[\mathrm{Fe} / \mathrm{H}]$ of $0.50 \pm 0.05$, using a spectrum with nearly twice the resolution as ours. Nevertheless, it is in good agreement with our 0.47 dex estimate with a line-to-line scatter of 0.11 dex derived using 30 lines.

For HD 104304 François (1988) found $[\mathrm{Fe} / \mathrm{H}]=0.16$ and $[\mathrm{S} / \mathrm{H}]=0.59$; our estimate of $[\mathrm{Fe} / \mathrm{H}]=0.17$ is in excellent agreement. We derive an $[\mathrm{S} / \mathrm{H}]$ value lower by $0.10 \mathrm{dex}$, but since our result is based on one fairly weak S I line, we consider this to be a good agreement.

Morell et al. (1992) derived $\mathrm{Fe}$ and $\mathrm{Th}$ abundances for a group of stars in order to estimate their ages. For HD 182572 and HD 196755 they derived $[\mathrm{Fe} / \mathrm{H}]=+0.3$ and +0.1 dex, respectively. This is in reasonable agreement with our results.

Edvardsson et al. (1993) analyzed 189 dwarf and subgiant stars with $[\mathrm{Fe} / \mathrm{H}]$ up to +0.25 dex, including HD 121370. The agreement between the two studies is very good, Table 4 . Also, the stellar parameters agree well. These different comparisons give us confidence that our analysis is satisfactory. 

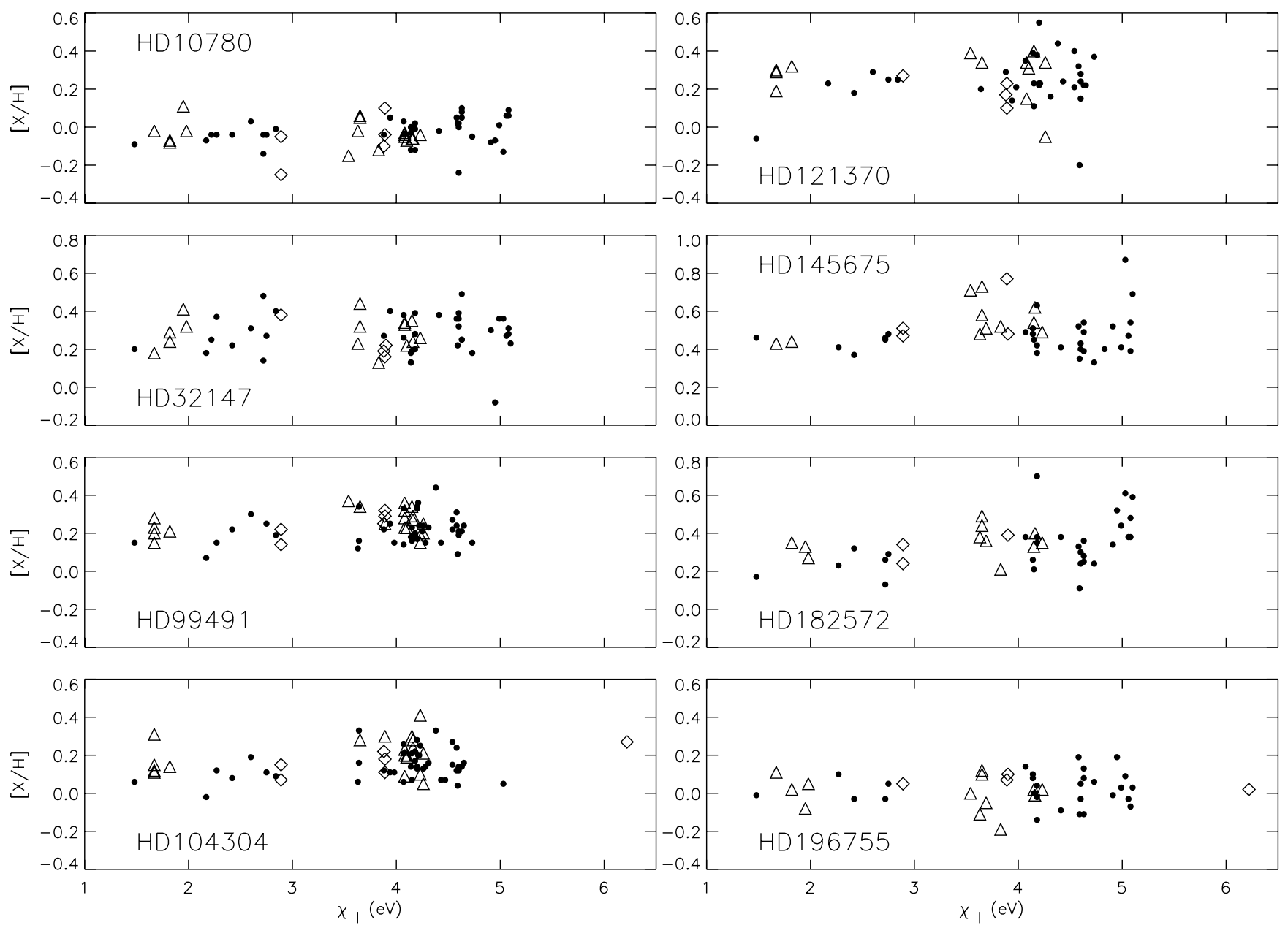

Fig. 4. Excitation equilibrium. Fe I lines are denoted by $\bullet$, Fe II by $\diamond$ and Ni I lines by $\triangle$. [X/H] denote the abundance relative to solar as derived from Fe I, Fe II and Ni I lines, respectively. Note the different ranges on the $y$-axes

\subsection{1. $\alpha$ Cen A and B}

As a final test of our analysis method and its compatibility with the analysis procedures adopted by other groups, we derived elemental abundances for the stars in the nearby triple system $\alpha$ Centauri from the equivalent widths published by Neuforge-Verheecke \& Magain (1997). They observed the two stars (components A and B) with the CATtelescope at La Silla with a resolution of 100000 and a final $S / N \sim 550$ and derived stellar abundances as well as stellar parameters self-consistently from the spectra. Using their published equivalent widths as well as their $\log g f$ and damping parameters with our set of model atmospheres and programs, we derive almost the same abundances for all elements with lines that are not affected by hyperfine splitting, see Table 5. In fact for most of those elements taking the hyperfine structure in the line profile into account makes very little difference in the derived abundances. This is true in particular for $\mathrm{Al}$.

For the A component we derive in general abundances 0.01 dex less than Neuforge-Verheecke \& Magain (1997) and for the B component 0.02-0.03 dex higher abundances, see Table 5. Iron is however 0.01 dex lower for the B component. We find this level of agreement satisfactory considering that we use model atmospheres of slightly different construction.

\section{Abundance results}

The stellar abundances derived in this study are summarized in Table 6 . We will discuss the abundance determination for each element separately. For some elements only one or a couple of lines have been used and the results are therefore more tentative than firm. The number of lines used for each element are also indicated in the table.

Iron abundances Iron abundances are derived from a large number of lines, 28 to 44 lines per star, which means that the errors in the mean are very small, typically less 0.02 dex. Thus, the error in Fe abundances is negligible in the error budget for the abundance ratios.

In Table 7 we compare the iron abundances in this study and those quoted by Taylor (1996). For HD 32147, HD 99491, HD 121370, HD 145675, and HD 182572 their 
Table 5. Comparison of abundances for $\alpha$ Cen A and B derived by Neuforge-Verheeke \& Magain (1997) and in this work using their equivalent widths and models as described in Sect. 4.5.1

\begin{tabular}{|c|c|c|c|c|c|c|}
\hline \multirow{3}{*}{ El. } & \multicolumn{3}{|c|}{$\alpha$ Cen A } & \multicolumn{3}{|c|}{$\alpha$ Cen B } \\
\hline & \# lines & This work & NVM97 & \# lines & This work & NVM97 \\
\hline & & {$[\mathrm{X} / \mathrm{H}] \pm \mathrm{sc}$} & {$[\mathrm{X} / \mathrm{H}] \pm$ error } & & {$[\mathrm{X} / \mathrm{H}] \pm \mathrm{sc}$} & {$[\mathrm{X} / \mathrm{H}] \pm$ error } \\
\hline O I & 3 & 0.200 .07 & 0.210 .06 & & & \\
\hline $\mathrm{Al} \mathrm{I}$ & 1 & 0.230 .00 & 0.240 .04 & 1 & 0.270 .00 & 0.240 .05 \\
\hline Si I & 3 & 0.260 .02 & 0.270 .03 & 3 & 0.300 .00 & 0.270 .04 \\
\hline $\mathrm{Ca} \mathrm{I}$ & 5 & 0.210 .05 & 0.220 .03 & 5 & 0.230 .05 & 0.210 .05 \\
\hline Sc II & 1 & 0.350 .00 & 0.250 .05 & 1 & 0.360 .00 & 0.260 .04 \\
\hline Ti I & 15 & 0.220 .04 & 0.250 .03 & 13 & 0.260 .07 & 0.270 .06 \\
\hline V I & 4 & 0.220 .04 & 0.230 .05 & 4 & 0.400 .07 & 0.320 .08 \\
\hline Cr I & 11 & 0.200 .03 & 0.240 .02 & 12 & 0.250 .03 & 0.270 .04 \\
\hline Cr II & 2 & 0.250 .02 & 0.260 .03 & 1 & 0.290 .00 & 0.260 .09 \\
\hline $\mathrm{Fe}$ I & 69 & 0.240 .06 & 0.250 .02 & 65 & 0.230 .05 & 0.240 .03 \\
\hline Fe II & 4 & 0.250 .03 & 0.250 .02 & 4 & 0.270 .04 & 0.250 .02 \\
\hline Co I & 3 & 0.290 .04 & 0.280 .04 & 3 & 0.390 .02 & 0.260 .04 \\
\hline $\mathrm{Ni} I$ & 26 & 0.290 .05 & 0.300 .03 & 25 & 0.310 .06 & 0.300 .02 \\
\hline Y II & 1 & 0.360 .00 & 0.200 .05 & 1 & 0.300 .00 & 0.140 .05 \\
\hline Eu II & 1 & 0.170 .00 & 0.150 .05 & 1 & 0.160 .00 & 0.140 .05 \\
\hline
\end{tabular}

Table 6. Derived elemental abundances for our program stars. The abundances are given in the format $[X / H]=\log (X / H)_{\star}-$ $\log (\mathrm{X} / \mathrm{H})_{\odot}$, where $\mathrm{X}$ denotes the element in question. The ionization stages are also indicated. The line-to-line scatter is given for each star and element when more than one line is used in the abundance analysis. The error in the derived abundance due to line-to-line scatter is thus = line-to-line scatter $/ \sqrt{n}_{\text {lines }}$, the number of lines used are given in parentheses. Oxygen abundances are given separately for the forbidden line at $630 \mathrm{~nm}$ and the triplet at $777 \mathrm{~nm}$. No star has observations of both

\begin{tabular}{|c|c|c|c|c|c|c|c|c|}
\hline & HD 10780 & HD 32147 & HD 99491 & HD 104304 & HD 121370 & HD 145675 & HD 182572 & HD 196755 \\
\hline $\mathrm{C}_{\mathrm{I}}$ & 0.28 & - & - & - & - & - & - & - \\
\hline $\mathrm{O}_{630}$ & - & - & 0.27 & 0.37 & - & - & - & - \\
\hline $\mathrm{O}_{777}$ & - & - & - & - & - & $0.480 .15(3)$ & $0.620 .10(3)$ & $0.110 .05(3)$ \\
\hline $\mathrm{NaI}$ & -0.03 & 0.64 & 0.34 & 0.37 & 0.50 & - & - & - \\
\hline $\mathrm{Al} \mathrm{I}$ & $-0.010 .03(3)$ & $0.480 .05(3)$ & 0.41 & 0.25 & - & $0.540 .02(3)$ & $0.550 .04(3)$ & $0.020 .05(3)$ \\
\hline Si I & $0.030 .05(5)$ & $0.360 .15(5)$ & $0.350 .10(7)$ & $0.270 .08(8)$ & $0.400 .14(6)$ & $\begin{array}{lll}0.61 & 0.07 & (3) \\
0.52 & 0.19 & (4)\end{array}$ & $0.490 .18(6)$ & $0.090 .05(4)$ \\
\hline Ca I & $0.130 .10(5)$ & - & 0.180 .08 (4) & 0.150 .07 (4) & 0.11 & 0.21 & 0.28 & $-0.020 .23(2)$ \\
\hline $\mathrm{S}_{\text {I }}$ & - & - & 0.56 & 0.49 & 0.72 & - & - & 0.38 \\
\hline $\mathrm{Sc} \mathrm{I}$ & - & - & 0.10 & 0.11 & - & - & - & - \\
\hline $\mathrm{Sc}$ II & -0.12 & 0.36 & - & $0.320 .13(3)$ & 0.11 & 0.66 & 0.36 & 0.11 \\
\hline Ti I & $0.100 .07(2)$ & $0.660 .18(2)$ & $0.170 .10(13)$ & $0.110 .10(12)$ & $0.22 \quad 0.22(3)$ & $0.620 .03(2)$ & $0.320 .03(3)$ & $0.120 .09(3)$ \\
\hline Cr I & $0.010 .15(3)$ & $0.500 .12(3)$ & $0.190 .11(6)$ & $0.140 .09(5)$ & $0.220 .04(2)$ & $0.420 .04(3)$ & $0.400 .01(2)$ & $-0.030 .16(2)$ \\
\hline Cr II & - & - & 0.35 & 0.26 & - & - & - & - \\
\hline Fe I & $-0.020 .07(39)$ & $0.280 .11(39)$ & $0.220 .08(42)$ & $0.150 .08(44)$ & $0.240 .14(32)$ & $0.470 .11(30)$ & $0.340 .14(29)$ & $0.020 .09(28)$ \\
\hline Fe II & -0.110 .10 & $0.240 .10(4)$ & $0.240 .07(5)$ & $0.170 .08(5)$ & $0.190 .07(4)$ & $\begin{array}{lll}0.49 & 0.02 & (3) \\
0.56 & 0.14 & (4)\end{array}$ & $0.320 .08(3)$ & $0.060 .03(4)$ \\
\hline Co I & -0.060 .06 & $0.560 .19(4)$ & $0.260 .08(5)$ & $0.040 .54(6)$ & 0.32 & $0.810 .11(4)$ & $0.470 .07(3)$ & 0.27 \\
\hline $\mathrm{Ni} I$ & $-0.030 .06(17)$ & $0.290 .08(15)$ & $0.26 \quad 0.07(20)$ & $0.200 .09(19)$ & $0.310 .16(13)$ & $0.550 .10(11)$ & $0.360 .08(18)$ & $0.000 .09(13)$ \\
\hline
\end{tabular}

SMR status is confirmed. HD 104304 is a marginal case and HD 10780 and HD 196755 are shown to not be SMR stars.

Oxygen The forbidden OI line at $6300 \AA$ was only measured in two stars, HD 99491 and HD 104304; they have $[\mathrm{O} / \mathrm{Fe}]$ of 0.05 and -0.17 dex, respectively. The errors in the $\mathrm{O}$ abundances are dominated by the measurement error for the [O I] line. Our spectra have $S / N$ of $\sim 250$, which translates into an uncertainty of $\sim 0.15$ dex in $[\mathrm{O} / \mathrm{Fe}]$. Thus, our observed $[\mathrm{O} / \mathrm{Fe}]$ are well within this scatter, and our data follow the trends found in Nissen \& Edvardsson (1992) and Feltzing \& Gustafsson (1998).

Three of our stars have useful observations of the triplet lines around $777 \mathrm{~nm}$. For those three stars we get $[\mathrm{O} / \mathrm{Fe}]=0.01,0.28$, and 0.09 dex respectively. Line-toline scatter is 0.1 dex or less for these stars which means that formal errors are less than 0.1 dex for all three stars. 
Table 7. Comparison of $[\mathrm{Fe} / \mathrm{H}]$ from Taylor (1996) and this work. We also give, in Cols. 3 and 4, the VSL and SMR status for the stars according to Taylor (1996)

\begin{tabular}{lllll}
\hline ID & VSL & SMR & $\begin{array}{l}{[\mathrm{Fe} / \mathrm{H}]} \\
(\text { Taylor })\end{array}$ & $\begin{array}{l}{[\mathrm{Fe} / \mathrm{H}]} \\
\text { This work }\end{array}$ \\
\hline HD 10780 & no & & 0.396 & -0.02 \\
HD 32147 & yes & & $>0.1$ & 0.28 \\
HD 99491 & marg & & 0.115 & 0.20 \\
HD 104304 & marg & & 0.326 & 0.16 \\
HD 121370 & & $95 \%$ & 0.305 & 0.25 \\
HD 145675 & marg & $98 \%$ & 0.38 & 0.47 \\
HD 182572 & & $98 \%$ & $>0.341$ & 0.35 \\
HD 196755 & & & 0.500 & 0.02 \\
\hline
\end{tabular}

These oxygen abundances should be fairly reliable as we are dealing with stars that are similar to the Sun and our study is differential. Edvardsson et al. (1993) found a good correlation between oxygen abundances derived from the forbidden line and those derived from the triplet. Note, however, that Feltzing \& Gustafsson (1998) found no such correlation for their very metal-rich sample. Thus, in conclusion, the $[\mathrm{O} / \mathrm{Fe}]$ for HD 196755 derived from the triplet should be robust while the $[\mathrm{O} / \mathrm{Fe}]$ for HD 145675 and HD 182572 are more uncertain in term of possible NLTE effects.

Sodium Only one Na I line was available for analysis in our spectra, but it has been widely used in other abundance studies. Therefore, we are confident that it is giving us reliable $\mathrm{Na}$ abundances.

Aluminum Aluminum shows a somewhat puzzling behaviour. Both Edvardsson et al. (1993) and Feltzing \& Gustafsson (1998) found $[\mathrm{Al} / \mathrm{Fe}]$ to be solar for all stars with $[\mathrm{Fe} / \mathrm{H}]>0.0$ dex. However, three of our stars show unexpectedly high $[\mathrm{Al} / \mathrm{Fe}]$ abundances. The line-to-line scatter is small for all stars with all three lines measured.

Silicon A flat distribution with some internal scatter is found. The line-to-line scatter is, as in Feltzing \& Gustafsson (1998), on the larger side for the number of lines used.

Calcium For three stars we observe several lines of CaI. These stars all show $[\mathrm{Ca} / \mathrm{Fe}]$ close to the solar value, as expected. For the other stars only one or two lines were available and the results are therefore uncertain.

Sulfur Our linelist contains $2 \mathrm{~S}$ I lines, however, for those stars where we could determine $\mathrm{S}$ abundances only one line was available in each star. Our abundances are therefore uncertain. We note that $[\mathrm{S} / \mathrm{Fe}]$ appears somewhat high.

Scandium Sc abundances were derived from both Sc I and $\mathrm{ScII}$ lines. The $[\mathrm{Sc} / \mathrm{Fe}]$ values fall within the range expected from Feltzing \& Gustafsson (1998) and the trend in our $[\mathrm{Sc} / \mathrm{Fe}]$ data is flat at around $0.1-0.15$ dex. We note though that our most metal rich stars are overabundant in Sc in contrast with Feltzing \& Gustafsson (1998) which show a tendency for the most metal-rich stars to be underabundant.

Titanium An overall flat trend is found for our stars in accordance with previous studies, i.e. Feltzing \& Gustafsson (1998). HD 32147 stands out with an extremely high Ti abundance, perhaps indicative of an underestimated temperature (see Thorén \& Feltzing 2000).

Chromium The behaviour is the same as found by Feltzing \& Gustafsson (1998), except for HD 32147. Essentially, $[\mathrm{Cr} / \mathrm{Fe}]$ has a flat trend above solar metallicity.

Cobalt We find a large scatter in the $[\mathrm{Co} / \mathrm{Fe}]$ abundances. The origin of this large scatter is not entirely clear, but it is accompanied by a large line-to-line scatter as well, and thus the reason may be sought among the selection of stellar lines.

Nickel A large number of Ni I lines were used, both to determine the effective temperature as well as the $\mathrm{Ni}$ abundances. All our stars have virtually solar $[\mathrm{Ni} / \mathrm{Fe}]$ in agreement with Edvardsson et al. (1993) and Feltzing \& Gustafsson (1998) for stars in this $[\mathrm{Fe} / \mathrm{H}]$ range. Interestingly, we find that our most metal-rich star, HD 145675, shows a slightly enhanced [Ni/Fe]. Tentative enhancements of $\mathrm{Ni}$ in the most metal-rich stars can be found also in the studies by Feltzing \& Gustafsson (1998) and Thorén \& Feltzing (2000). All three studies adopted different techniques for derivation of stellar parameters, and thus the result seems to be significant. However, further investigations should be undertaken.

\section{Ages of SMR stars and the Age-metallicity relation}

An age-metallicity relation among dwarf stars in the solar neighbourhood is a key observable that models of galactic chemical evolution must match. The most important recent studies include Edvardsson et al. (1993), Carraro et al. (1998), and Rocha-Pinto et al. (2000). The first two studies use the same $[\mathrm{Fe} / \mathrm{H}]$, as derived in Edvardsson et al. (1993) from detailed abundance analysis. Carraro et al. (1998) make use of the age determinations done for Edvardsson et al. (1993) sample post Hipparcos ( $\mathrm{Ng}$ \& Bertelli 1998). Essentially, their data show a declining trend such that more metal-poor stars are older. However, the intrinsic scatter appears large in both age and $[\mathrm{Fe} / \mathrm{H}]$ and a unique age-metallicity relation may not be present. The study by Rocha-Pinto et al. (2000) used a different technique to determine ages, chromospheric activity. They arrive at the conclusion that there exists a unique age-metallicity relation in the solar neighbourhood. 


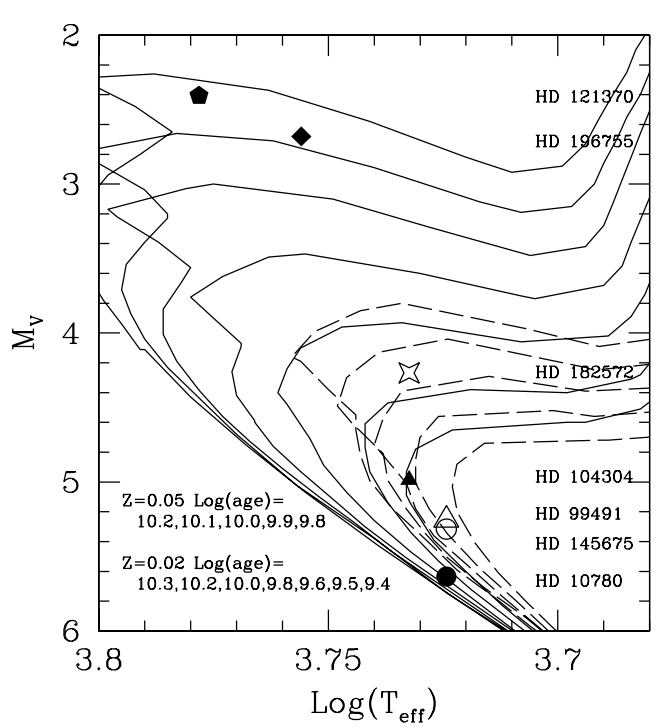

Fig. 5. Diagram used to estimate ages for our program stars. Isochrones are from Bertelli et al. (1994). Full curves correspond to $Z=0.02$, and the dashed curves to $Z=0.05$ isochrones, respectively. Filled symbols denote stars with $[\mathrm{Fe} / \mathrm{H}] \leq 0.20$ dex and open circles stars with $[\mathrm{Fe} / \mathrm{H}]>0.20$ dex. HD 32147 is too cool to show on the diagram

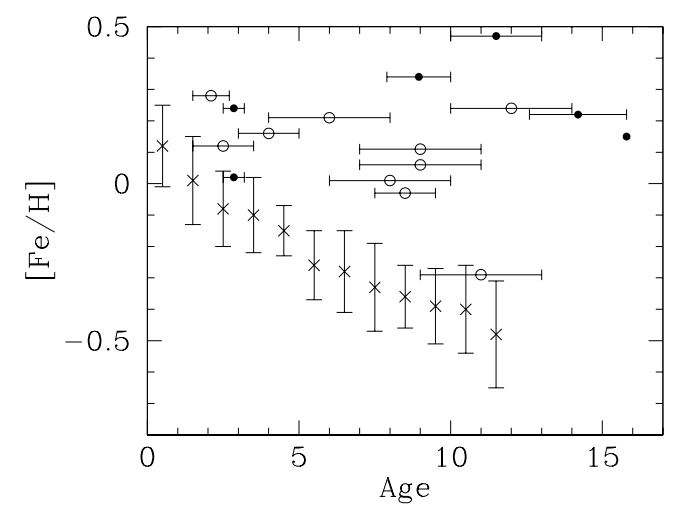

Fig. 6. A comparison of our isochrone ages with the general age-metallicity relation derived by Rocha-Pinto et al. (2000). - refer to stars in this study, o to stars from Gonzalez, see Table $8 . \times$ refers to the age-metallicity relation from RochaPinto et al. where the error bars give the $1 \sigma$ scatter around the mean $[\mathrm{Fe} / \mathrm{H}]$ for each age bin

The scatter in both age and metallicity are found to be small for all ages and metallicities (see their Fig. 13).

SMR stars are rare and therefore none of the studies discussed contain large numbers of them, in fact e.g. the Edvardsson et al. (1993) sample was selected with an upper limit in metallicity near 0.2 dex. Such a bias is not present in the Rocha-Pinto et al. (2000) sample, and they have a few stars of up to $\sim 0.3$ dex (their Fig. 13). It is therefore valuable to derive ages for our small sample of stars and compare them to that of the general agemetallicity relations found in previous studies.

We have simply estimated the ages of the stars by plotting them in the $M_{V}-T_{\text {eff }}$ plane and using the Bertelli et al. (1994) isochrones, Fig. 5. The ages were estimated by eye. The correct isochrones were chosen depending on the $[\mathrm{Fe} / \mathrm{H}]$ for each star as derived in this study. In order to see if the age-metallicity relation appears unique also for the most metal-rich stars, we compare our data and the ages from the several papers by Gonzalez and co-workers, see Table 8 , with the age-metallicity relation found in Rocha-Pinto et al. (2000) in Fig. 6.

A possible error source in the age determination of SMR stars is the presence of planets. Gonzalez (1998) noted that if one or several planets have been engulfed by a star, then its $[\mathrm{Fe} / \mathrm{H}]$ may increase by up to around 0.10 dex. If this has happened, then the abundances and age for a polluted star will no longer represent it's true age and abundances. However, such a change in metallicity would still not turn a $\sim 10$ Gyr star into a star of only a few Gyr, as required to fit into a general age-metallicity relation.

We note that our sample is not complete or in any other way well-defined. However, it proves that there also exist stars that are both very old and at the same time very metal-rich, also taking the errors in the ages into account. This casts doubts on the possibility of defining a one-to-one relation between age and metallicity among the solar neighbourhood stars.

\section{Velocity data}

Spatial velocity data were calculated using the Hipparcos parallaxes and proper motions. Radial velocities were taken from Barbier-Brossat et al. (1994). For our stars the uncertainties in the parallaxes are small, less than $3 \%$ of the parallax, Table 1. Data were also obtained for the stars from the Gonzalez (1999) compilation. The velocities are presented in Table 8 . Note that we here quote the velocities relative to the local standard of rest (LSR) and Gonzalez (1999) quoted velocities relative to the sun.

From Table 8 we see that all the stars have $W$ velocities well below the $\sigma_{W}$ of the general population of stars with similar $B-V$. Figure 5 in Dehnen \& Binney (1998) illustrates how $\sigma_{U, V, W}$ varies with $B-V$. Also, most of the stars in Table 8 have both $V$ and $U$-velocities well below $1 \sigma$ for the general population. We have quantified this by calculating the probabilities that any one of our stars belongs to either the thin or the thick disk by using a model where $94 \%$ of the solar neighbourhood stars belong to a thin disk with $\sigma_{U}=35, \sigma_{V}=25$, and $\sigma_{W}=17 \mathrm{~km} \mathrm{~s}^{-1}$ and the remaining $6 \%$ to the thick disk with $\sigma_{U}=70, \sigma_{V}=50$, and $\sigma_{W}=34 \mathrm{~km} \mathrm{~s}^{-1}$. Only one of our stars, HD 182572, has a probability that it belongs to the thick disk larger than that it should belong to the thin. We estimate that, given the galactic model, this star has $75 \%$ chance of belonging to the thick disk. Thus, we conclude that our SMR and planet-bearing stars samples the thin disk.

Fuhrmann (1998) found that stars with thick disk kinematics were enhanced in $[\mathrm{Mg} / \mathrm{Fe}]$ as compared to thin disk stars at the same metallicity. We have not measured $\mathrm{Mg}$ lines in our spectra. We did, however, 
Table 8. Velocity data and age determinations. The top half of the table contains the stars in this study and the lower section stars from Gonzalez (1999) and Gonzalez \& Laws (2000). Age estimates, using isochrones, from this study are given in Col. 6 top half. The age estimates for the stars in the second section are also based on the Bertelli et al. (1994) stellar isochrones, with typical uncertainties of 1 to 2 Gyrs. In Col. 7 we reproduce the Ca II activity index ages from Gonzalez (1998). In the last column we give $[\mathrm{Fe} / \mathrm{H}]$ as determined in this study or from Gonzalez (1999) and Gonzalez \& Laws (2000)

\begin{tabular}{|c|c|c|c|c|c|c|c|}
\hline ID & Name & $\begin{array}{r}U_{\mathrm{LSR}} \\
\left(\mathrm{km} \mathrm{s}^{-1}\right)\end{array}$ & $\begin{array}{r}V_{\mathrm{LSR}} \\
\left(\mathrm{kms}^{-1}\right)\end{array}$ & $\begin{array}{r}W_{\mathrm{LSR}} \\
\left(\mathrm{km} \mathrm{s}^{-1}\right)\end{array}$ & $\begin{array}{r}\text { Age (isochrones) } \\
(\mathrm{Gyr})\end{array}$ & $\begin{array}{r}\text { Age (Ca II) } \\
\text { (Gyr) }\end{array}$ & {$[\mathrm{Fe} / \mathrm{H}]$} \\
\hline HD 10780 & & -14.8 & -11.3 & 1.6 & - & & -0.02 \\
\hline HD 32147 & & 10.7 & -51.8 & -6.3 & - & & 0.28 \\
\hline HD 99491 & & -49.8 & -4.1 & -8.7 & $12.6-15.8$ & & 0.22 \\
\hline HD 104304 & & 31.6 & -10.2 & -9.2 & $\leq 15.8$ & & 0.15 \\
\hline HD 121370 & & 19.2 & -12.0 & 4.6 & $2.5-3.2$ & & 0.24 \\
\hline HD 145675 & 14 Her & 35.6 & -2.0 & -2.9 & $10-13.0$ & & 0.47 \\
\hline HD 182572 & & -106.7 & -25.2 & -13.5 & $7.9-10.0$ & & 0.34 \\
\hline HD 196755 & & -48.1 & 29. & -11.7 & $2.5-3.2$ & & 0.02 \\
\hline HD 9826 & $v$ And & 4.3 & -34.1 & 0.6 & 2.7 & & 0.12 \\
\hline HD 75732 & $\rho^{1} \mathrm{Cnc}$ & -27.3 & -13.2 & -0.9 & & 5 & 0.45 \\
\hline HD 75289 & & 31.1 & -12.4 & -14.5 & 2.1 & & 0.28 \\
\hline HD 95128 & $47 \mathrm{UMa}$ & -14.7 & 2.6 & 8.8 & 6.3 & 7 & 0.01 \\
\hline HD 117176 & 70 Vir & 23.2 & -46.9 & 3.4 & 8 & 9 & -0.03 \\
\hline HD 120136 & $\tau$ Boo & -23.5 & -13.8 & 0.3 & 1 & & 0.32 \\
\hline HD 143761 & $\rho \mathrm{CrB}$ & 64.1 & -30.7 & 28.5 & 12.3 & & -0.29 \\
\hline HD 186408 & 16 Cyg A & 27.6 & -23.6 & 7.2 & 9.0 & & 0.11 \\
\hline HD 186427 & 16 Cyg B & 27.1 & -24.7 & 5.4 & 9.0 & 7 & 0.06 \\
\hline HD 187123 & & 11.6 & -10.6 & -36.4 & 5.5 & & 0.16 \\
\hline HD 217014 & $51 \mathrm{Peg}$ & -5.6 & -24.2 & 22.3 & 6.0 & 10 & 0.21 \\
\hline HD 210277 & & 12.4 & -46.8 & 3.0 & 8.5 & & 0.24 \\
\hline
\end{tabular}

measure Si, and our abundance result for HD 182572 gives $[\mathrm{Si} / \mathrm{Fe}]=0.16 \pm 0.09$. Compared with the general trend of $[\mathrm{Si} / \mathrm{Fe}]$ for metal-rich stars in Feltzing \& Gustafsson (1998), this is above the mean; however, their data exhibit a large scatter. We have also determined Ca abundances for this star, however, only one line was available. This line seems to give fairly low $\mathrm{Ca}$ abundances in all of the stars with more than two lines observed and may thus be underestimating the true $\mathrm{Ca}$ abundance in this star. Note that it is not inconsistent that we also find thin disk stars with the same Si abundance as, if HD 182572 is a thick disk star, then it might be showing us the abundance trend after the decline in $[\mathrm{X} / \mathrm{Fe}]$, where $\mathrm{X}$ is either $\mathrm{O}$ or an $\alpha$ element, sets in due to increasing relative contribution of SNIa.

\section{SMR - planet connection}

Several of the nearby SMR star candidates listed by Taylor (1996, Table 4) have been found to harbour planets. In particular, the planet-bearing stars HD 75732, HD 145675 , and HD 217014 are included in Taylor's list of 29 SMR class IV-V star candidates. Not all the stars in his list have been searched for planets as of yet, so the fraction of SMR star candidates with planets may increase. Butler et al. (2000) provide independent confirming evidence for a preponderance of planets among metal-rich stars; they note that of their 600 Keck targets, half of which are metal-poor, 2 planets have been found around metal-poor stars and 10 around metal-rich stars. Also, several planetbearing stars not in Taylor's list have recently been found to be likely SMR stars. Examples in this group include HD 120136, HD 217107, and HD 210277 (Gonzalez 2000). It appears that the Doppler planet search method is also an efficient detector of new SMR star candidates!

Gonzalez et al. (1999) suggested that BD $-10^{\circ} 3166$ be searched for planets on the basis of its similarity to HD 75732 and HD 145675 (similar $T_{\text {eff }}$ and [Fe/H]). Butler et al. (2000) reported on the detection of a planet around $\mathrm{BD}-10^{\circ} 3166$, which they had placed on their monitoring program as a result of Gonzalez et al.'s suggestion. Another star, HD 89744, was suggested to Geoff Marcy as a planet-bearing candidate by one of us (G.G.) on the basis of its high $[\mathrm{Fe} / \mathrm{H}]$ and low $[\mathrm{C} / \mathrm{Fe}]$. Sylvain et al. (2000) announced the discovery of a planet around this star (note: they began observing this star about 2 years before Gonzalez's prediction). The successful prediction of the presence of planets around two stars provides strong independent confirming evidence of the planet - SMR star connection. The low $[\mathrm{C} / \mathrm{Fe}]$ values seen among stars with 
planets is the first convincing evidence of a trend with abundance ratios. The physical significance of this trend is not yet known.

\section{Conclusions}

We have presented detailed abundance analyses at high resolution for 8 possible SMR dwarf stars and subgiants. Four of these stars have previously been studied at high resolution; our results in general agree well with them. For the remaining four stars this is, to the best of our knowledge, the first study of this sort.

We find in particular that:

- HD 32147, HD 99491, HD 121370, HD 145675, HD 182572 all have $[\mathrm{Fe} / \mathrm{H}] \geq 0.2$ dex, the lower limit for super metal rich status as defined by Taylor (1996);

- HD 104304 presents a marginal case;

- HD 10780 and HD 196755 are found to have solar iron abundances and are thus not SMR stars;

- some metal-rich, and in particular SMR, stars are old, showing that the large scatter in $[\mathrm{Fe} / \mathrm{H}]$ at a given age among nearby solar type stars exists at all ages and that a one-to-one relation between age and metallicity among the solar neighbourhood stars may not exist for all metallicities;

- that metal-rich stars are mainly confined to the galactic plane, however, one star in our sample appears to be a thick disk candidate of extremely high metallicity;

- there exists a correlation between SMR-ness and the presence of planets.

Further investigations should be undertaken to prove the possibility that $[\mathrm{Ni} / \mathrm{Fe}]$ is starting to increase at the highest metallicities as well as the very real possibility that there exists extreme thick disk stars with very high metallicities. In particular, $\alpha$-element abundances should be carefully studied for such candidates.

Acknowledgements. SF acknowledges financial support from the Swedish Natural Research Council under their postdoc program. GG acknowledges financial support from the Kennilworth Fund of the New York Community Trust.

Johan Holmberg at Lund Observatory is thanked for providing stellar velocities.

\section{References}

Alonso, A., Arribas, S., \& Martinez-Roger, C. 1996, A\&A, 313, 873

Asplund, M., Gustafsson, B., Kiselman, D., \& Eriksson, K. 1997, A\&A, 318, 521

Barbier-Brossat, M., Petit, M., \& Figon, P. 1994, A\&AS, 108, 603

Barbuy, B., \& Grenon, M. 1990, in ESO/CTIO Workshop on Bulges of Galaxies, 83

Bertelli, G., Bressan, A., Chiosi, C., Fagotto, F., \& Nasi, E. 1994, A\&AS, 106, 275

Blackwell, D. E., Lynas-Gray, A. E., \& Smith, G. 1995, A\&A, 296,217
Butler, R. P., Vogt, S. S., Marcy, G. W., et al. 2000, ApJ, submitted

Carraro, G., Ng, Y. K., \& Protinari, L. 1998, MNRAS, 296, 1045

Castro, S., Rich, R. M., Grenon, M., Barbuy, B., \& McCarthy, J. K. 1997, AJ, 114, 376

Cayrel de Strobel, G., Soubiran, C., Friel, E. D., Ralite, N., \& François, P. 1997, A\&AS, 124, 299

Dehnen, W., \& Binney, J. J. 1998, MNRAS, 298, 387

Edvardsson, B., Andersen, J., Gustafsson, B., et al. 1993, A\&A, 275,101

ESA, 1997, The Hipparcos and Tycho Catalogues, ESA SP1200

Feltzing, S., \& Gustafsson, B. 1998, A\&AS, 129, 237

François, P. 1988, A\&A, 195, 226

Fuhrmann, K. 1998, A\&A, 338, 161

Fuhrmann, K., Pfeiffer, M. C., \& Bernkopf, J. 1997, A\&A, 326, 1081

Fuhrmann, K., Pfeiffer, M. C., \& Bernkopf, J. 1998, A\&A, 336, 942

Gonzalez, G. 1998, A\&A, 334, 221

Gonzalez, G. 1999, MNRAS, 308, 447

Gonzalez, G. 2000, in ASP Conf. Ser., Disks, Planetesimals, and Planets, ed. F. Garzon, C. Eiroa, D. de Winter, \& T. J. Mahoney, in press

Gonzalez, G., \& Lambert, D. L. 1996, AJ, 111, 424

Gonzalez, G., \& Laws, C. 2000, AJ, 119, 390

Gonzalez, G., Wallerstein, G., \& Saar, S. H. 1999, ApJ, 511, L111

Gronbech, S. L., \& Olsen, E. H. 1977, A\&AS, 27, 443

Gustafsson, B., Bell, R. A., Eriksson, K., \& Nordlund, Å. 1975, A\&A, 42, 407

Hannaford, P., Lowe, R. M., Grevesse, N., \& Noels, A. 1992, A\&A, 259, 301

Holweger, H., Bard, A., Kock, A., \& Kock, M. 1991, A\&A, 249, 545

Kurucz, R. L., Furenlid, I., Brault, J., \& Testerman, L. 1984, Solar Flux Atlas from 296 to 1300 nm, National Solar Observatory

Mäckle, R., Holweger, H., Griffin, R., \& Griffin, R. 1975, A\&A, 38,239

McCarthy, J. K., Sandiford, B. A., Boyd, D., \& Booth, J. 1993, PASP, 105, 881

McWilliam, A., \& Rich, M. 1994, ApJS, 91, 749

Morell, O., Källander, D., \& Butcher, H. R. 1992, A\&A, 259, 543

Neuforge, C. 1992, in Origin and Evolution of Elements, ed. N. Prantzos, E. Vangioni-Flam, \& M. Cassé, 63

Neuforge-Verheecke, C., \& Magain, P. 1997, A\&A, 328, 261

Ng, Y. K., \& Bertelli, G. 1998, A\&A, 329, 943

Nissen, P. E., \& Edvarsson, B. 1992, A\&A, 261, 255

Olsen, E. H. 1984, A\&AS, 57, 4430

Olsen, E. H. 1993, A\&AS, 102, 89

Olsen, E. H. 1994a, A\&AS, 104, 429

Olsen, E. H., 1994b, A\&AS, 106, 257

Rocha-Pinto, H. J., Maciel, W. J., Scalo, J., \& Flynn, C. 2000, A\&A, 358, 850

Smith, G., \& Ruck, M. J. 2000, A\&A, 356, 570

Spinrad, H., \& Taylor, B. J. 1969, ApJ, 157, 1279

Sylvain, G. K., Brown, T. M., Fischer, D. A., Nisenson, P., \& Noyes, R. W. 2000, ApJ, 533, L147

Taylor, B. J. 1994, PASP, 106, 600

Taylor, B. J. 1996, ApJS, 102, 105

Thorén, P., \& Feltzing, S. 2000, A\&A, 363, 692 
Table A1. Line data

\begin{tabular}{|c|c|c|c|c|c|}
\hline $\begin{array}{c}\lambda \\
{[\AA]}\end{array}$ & $\begin{array}{r}\chi_{l} \\
{[\mathrm{eV}]}\end{array}$ & $\log g f$ & $\delta \Gamma_{6}$ & $\begin{array}{c}\Gamma_{\mathrm{rad}} \\
{\left[\mathrm{s}^{-1}\right]}\end{array}$ & Note \\
\hline \multicolumn{6}{|c|}{$\mathbf{C}_{\mathrm{I}} ; \log \epsilon_{\odot}=8.56$} \\
\hline 6587.61 & 8.53 & -1.246 & 2.50 & $1.00 \mathrm{e}+08$ & \\
\hline \multicolumn{6}{|c|}{$\mathbf{O}_{\mathrm{I}} ; \log \epsilon_{\odot}=8.93$} \\
\hline 6300.310 & 0.00 & -9.75 & 2.50 & $1.00 \mathrm{e}+08$ & \\
\hline 7771.95 & 9.14 & 0.26 & 2.50 & $1.00 \mathrm{e}+08$ & \\
\hline 7774.17 & 9.14 & 0.12 & 2.50 & $1.00 \mathrm{e}+08$ & \\
\hline 7775.35 & 9.14 & -0.11 & 2.50 & $1.00 \mathrm{e}+08$ & \\
\hline \multicolumn{6}{|c|}{$\mathbf{N a}$ I; $\log \epsilon_{\odot}=6.33$} \\
\hline 6154.23 & 2.10 & -1.58 & 2.10 & $7.08 \mathrm{e}+07$ & \\
\hline \multicolumn{6}{|c|}{ Al I; $\log \epsilon_{\odot}=6.47$} \\
\hline 6698.67 & 3.143 & -1.89 & 2.50 & $3.02 \mathrm{e}+08$ & \\
\hline 7835.30 & 4.022 & -0.78 & 2.50 & $7.94 \mathrm{e}+07$ & \\
\hline 7836.13 & 4.022 & -0.60 & 2.50 & $7.94 \mathrm{e}+07$ & \\
\hline \multicolumn{6}{|c|}{ Si I $; \log \epsilon_{\odot}=7.55$} \\
\hline 5622.22 & 4.930 & -2.95 & 2.50 & $1.95 \mathrm{e}+08$ & \\
\hline 5665.55 & 4.920 & -2.02 & 2.50 & $1.95 \mathrm{e}+08$ & \\
\hline 5793.07 & 4.930 & -1.95 & 2.50 & $1.95 \mathrm{e}+08$ & \\
\hline 6125.02 & 5.614 & -1.55 & 2.50 & $1.00 \mathrm{e}+08$ & \\
\hline 6142.48 & 5.619 & -1.50 & 2.50 & $1.00 \mathrm{e}+08$ & \\
\hline 6155.69 & 5.619 & -2.43 & 2.50 & $1.00 \mathrm{e}+08$ & \\
\hline 6237.31 & 5.614 & -1.15 & 2.50 & $1.00 \mathrm{e}+08$ & \\
\hline 6721.84 & 5.863 & -1.16 & 2.50 & $1.00 \mathrm{e}+08$ & \\
\hline 6741.62 & 5.984 & -1.63 & 2.50 & $2.69 \mathrm{e}+07$ & \\
\hline 6848.58 & 5.863 & -1.65 & 2.50 & $1.00 \mathrm{e}+08$ & \\
\hline 7455.37 & 5.964 & -2.00 & 2.50 & $1.00 \mathrm{e}+08$ & \\
\hline 7760.62 & 6.206 & -1.36 & 2.50 & $1.00 \mathrm{e}+08$ & \\
\hline 7799.99 & 6.181 & -0.77 & 2.50 & $1.00 \mathrm{e}+08$ & \\
\hline \multicolumn{6}{|c|}{$\mathbf{S}_{\mathrm{I}} ; \log \epsilon_{\odot}=7.21$} \\
\hline 6743.531 & 7.866 & -0.84 & 2.50 & $3.80 \mathrm{e}+07$ & \\
\hline 6757.171 & 7.870 & -0.53 & 2.50 & $3.89 \mathrm{e}+07$ & \\
\hline \multicolumn{6}{|c|}{$\mathbf{C a} \mathrm{I} ; \log \epsilon_{\odot}=6.36$} \\
\hline 5512.980 & 2.933 & -0.66 & 2.50 & $2.80 \mathrm{e}+08$ & \\
\hline 5581.965 & 2.523 & -0.87 & 2.50 & $7.13 e+07$ & \\
\hline 5867.562 & 2.933 & -1.61 & 2.50 & $2.62 \mathrm{e}+08$ & \\
\hline 6166.439 & 2.521 & -1.22 & 2.50 & $1.86 \mathrm{e}+07$ & \\
\hline 6169.042 & 2.523 & -0.90 & 2.50 & $1.86 \mathrm{e}+07$ & \\
\hline 6169.563 & 2.526 & -0.67 & 2.50 & $1.88 \mathrm{e}+07$ & \\
\hline 6455.598 & 2.523 & -1.48 & 2.50 & $4.64 \mathrm{e}+07$ & \\
\hline 6464.673 & 2.526 & -2.36 & 2.50 & $4.64 \mathrm{e}+07$ & \\
\hline 6471.662 & 2.526 & -0.98 & 2.50 & $4.42 \mathrm{e}+07$ & \\
\hline \multicolumn{6}{|c|}{ Sc I $; \log \epsilon_{\odot}=3.10$} \\
\hline 5484.626 & 1.851 & 0.37 & 1.50 & $1.46 \mathrm{e}+08$ & \\
\hline \multicolumn{6}{|c|}{ Sc II; $\log \epsilon_{\odot}=3.10$} \\
\hline 5526.790 & 1.768 & 0.09 & 1.50 & $2.16 \mathrm{e}+08$ & \\
\hline 6300.698 & 1.507 & -2.01 & 1.50 & $2.31 \mathrm{e}+08$ & \\
\hline 6604.601 & 1.357 & -1.16 & 1.50 & $1.46 \mathrm{e}+08$ & \\
\hline \multicolumn{6}{|c|}{$\mathbf{T i} \mathrm{I} ; \log \epsilon_{\odot}=4.99$} \\
\hline 5490.148 & 1.460 & -0.98 & 2.50 & $1.43 e+08$ & \\
\hline 5739.469 & 2.249 & -0.78 & 2.50 & $6.60 \mathrm{e}+07$ & \\
\hline 5739.978 & 2.236 & -0.74 & 2.50 & $6.53 \mathrm{e}+07$ & \\
\hline 5823.686 & 2.267 & -1.05 & 2.50 & $6.53 e+07$ & \\
\hline 5832.473 & 3.337 & -0.28 & 2.50 & $1.45 \mathrm{e}+08$ & \\
\hline 5866.451 & 1.067 & -0.83 & 2.50 & $4.40 \mathrm{e}+08$ & \\
\hline 6091.171 & 2.267 & -0.43 & 2.50 & $8.50 \mathrm{e}+07$ & \\
\hline 6092.792 & 1.887 & -1.45 & 2.50 & $1.27 \mathrm{e}+08$ & \\
\hline 6098.658 & 3.062 & -0.07 & 2.50 & $5.43 e+07$ & \\
\hline 6126.216 & 1.067 & -1.41 & 2.50 & $9.93 e+06$ & \\
\hline 6599.105 & 0.900 & -2.07 & 2.50 & $1.22 \mathrm{e}+06$ & \\
\hline 6743.122 & 0.900 & -1.73 & 2.50 & $6.93 e+05$ & \\
\hline 6745.547 & 2.236 & -1.36 & 2.50 & $1.44 \mathrm{e}+08$ & \\
\hline
\end{tabular}

Table A1. continued

\begin{tabular}{|c|c|c|c|c|c|}
\hline $\begin{array}{c}\lambda \\
{[\AA]} \\
\AA\end{array}$ & $\begin{array}{r}\chi_{l} \\
{[\mathrm{eV}]}\end{array}$ & $\log g f$ & $\delta \Gamma_{6}$ & $\begin{array}{l}\Gamma_{\mathrm{rad}} \\
{\left[\mathrm{s}^{-1}\right]}\end{array}$ & Note \\
\hline \multicolumn{6}{|c|}{$\mathbf{V} \mathrm{I} ; \log \epsilon_{\odot}=4.00$} \\
\hline 5670.853 & 1.081 & -0.46 & 2.50 & $5.23 \mathrm{e}+06$ & \\
\hline 5727.048 & 1.081 & -1.22 & 2.50 & $7.57 \mathrm{e}+08$ & \\
\hline 5727.652 & 1.051 & -0.90 & 2.50 & $6.15 \mathrm{e}+07$ & \\
\hline 5830.675 & 3.113 & 0.61 & 2.50 & $1.83 \mathrm{e}+08$ & \\
\hline 6039.722 & 1.064 & -0.72 & 2.50 & $3.98 \mathrm{e}+07$ & \\
\hline 6090.214 & 1.081 & -0.15 & 2.50 & $3.98 \mathrm{e}+07$ & \\
\hline 6111.645 & 1.043 & -0.795 & 2.50 & $3.90 \mathrm{e}+07$ & \\
\hline 6135.361 & 1.051 & -0.766 & 2.50 & $3.90 \mathrm{e}+07$ & \\
\hline 6150.157 & 0.301 & -1.54 & 2.50 & $7.81 \mathrm{e}+05$ & \\
\hline 6199.197 & 0.287 & -1.48 & 2.50 & $3.25 \mathrm{e}+06$ & \\
\hline 6224.529 & 0.287 & -1.84 & 2.50 & $1.22 \mathrm{e}+06$ & \\
\hline 6251.827 & 0.287 & -1.45 & 2.50 & $3.07 \mathrm{e}+07$ & \\
\hline 6256.887 & 0.275 & -2.17 & 2.50 & $2.94 \mathrm{e}+06$ & \\
\hline 6452.341 & 1.195 & -0.836 & 2.50 & $3.99 \mathrm{e}+07$ & \\
\hline \multicolumn{6}{|c|}{ Cr I; $\log \epsilon_{\odot}=5.67$} \\
\hline 5628.621 & 3.422 & -0.83 & 2.50 & $6.52 \mathrm{e}+07$ & \\
\hline 5664.555 & 3.826 & -0.87 & 2.50 & $4.80 \mathrm{e}+07$ & \\
\hline 5783.886 & 3.322 & -0.26 & 2.50 & $9.98 \mathrm{e}+07$ & \\
\hline 5787.965 & 3.322 & -0.21 & 2.50 & $1.00 \mathrm{e}+08$ & \\
\hline 6630.005 & 1.030 & -3.46 & 2.50 & $2.40 \mathrm{e}+07$ & \\
\hline 6669.255 & 4.175 & -0.47 & 2.50 & $3.66 \mathrm{e}+07$ & \\
\hline 7400.226 & 2.900 & -0.171 & 2.50 & $6.76 \mathrm{e}+07$ & \\
\hline \multicolumn{6}{|c|}{ Cr II; $\log \epsilon_{\odot}=5.67$} \\
\hline 5502.067 & 4.168 & -1.99 & 2.50 & $2.55 \mathrm{e}+07$ & \\
\hline \multicolumn{6}{|c|}{$\mathbf{F e} \mathrm{I} ; \log \epsilon_{\odot}=7.51$} \\
\hline 5491.832 & 4.186 & -2.14 & 2.00 & $1.44 \mathrm{e}+08$ & \\
\hline 5494.463 & 4.076 & -1.85 & 2.00 & $2.90 \mathrm{e}+07$ & \\
\hline 5522.447 & 4.209 & -1.43 & 2.00 & $8.97 \mathrm{e}+07$ & \\
\hline 5539.280 & 3.642 & -2.49 & 2.00 & $2.60 \mathrm{e}+07$ & \\
\hline 5543.936 & 4.217 & -1.10 & 2.00 & $2.39 \mathrm{e}+08$ & \\
\hline 5560.212 & 4.434 & -1.09 & 2.00 & $1.64 \mathrm{e}+08$ & \\
\hline 5577.030 & 5.033 & -1.45 & 2.00 & $6.89 \mathrm{e}+08$ & \\
\hline 5579.340 & 4.231 & -2.29 & 2.00 & $2.55 \mathrm{e}+08$ & \\
\hline 5607.664 & 4.154 & -2.18 & 2.00 & $3.50 \mathrm{e}+08$ & \\
\hline 5608.972 & 4.209 & -2.31 & 2.00 & $8.89 \mathrm{e}+07$ & \\
\hline 5611.360 & 3.635 & -2.91 & 2.00 & $1.25 \mathrm{e}+08$ & \\
\hline 5618.633 & 4.209 & -1.34 & 2.00 & $1.05 \mathrm{e}+08$ & \\
\hline 5619.595 & 4.386 & -1.48 & 2.00 & $1.78 \mathrm{e}+08$ & \\
\hline 5636.696 & 3.640 & -2.56 & 2.00 & $3.90 \mathrm{e}+07$ & \\
\hline 5638.262 & 4.220 & -0.88 & 2.00 & $1.94 \mathrm{e}+08$ & \\
\hline 5646.684 & 4.260 & -2.40 & 2.00 & $1.00 \mathrm{e}+08$ & \\
\hline 5651.469 & 4.473 & -1.76 & 2.00 & $1.62 \mathrm{e}+08$ & \\
\hline 5652.010 & 4.218 & -1.82 & 2.00 & $2.36 \mathrm{e}+08$ & \\
\hline 5741.848 & 4.256 & -1.66 & 2.00 & $2.11 \mathrm{e}+08$ & \\
\hline 5793.689 & 4.593 & -1.29 & 2.00 & $5.38 \mathrm{e}+07$ & \\
\hline 5811.914 & 4.143 & -2.38 & 2.00 & $3.76 \mathrm{e}+07$ & \\
\hline 5814.807 & 4.283 & -1.84 & 2.00 & $2.11 \mathrm{e}+08$ & \\
\hline 5852.219 & 4.548 & -1.21 & 2.00 & $1.90 \mathrm{e}+08$ & \\
\hline 5855.077 & 4.608 & -1.54 & 2.00 & $1.91 \mathrm{e}+08$ & \\
\hline 6034.035 & 4.312 & -2.30 & 2.00 & $1.59 \mathrm{e}+08$ & \\
\hline 6089.580 & 4.580 & -1.30 & 2.00 & $9.27 \mathrm{e}+07$ & \\
\hline 6094.374 & 4.652 & -1.58 & 2.00 & $1.92 \mathrm{e}+08$ & \\
\hline 6096.665 & 3.984 & -1.81 & 2.00 & $4.53 \mathrm{e}+07$ & \\
\hline 6105.131 & 4.548 & -1.91 & 2.00 & $1.00 \mathrm{e}+08$ & \\
\hline 6151.618 & 2.176 & -3.329 & 2.00 & $1.55 \mathrm{e}+08$ & \\
\hline 6157.728 & 4.076 & -1.28 & 2.00 & $5.02 \mathrm{e}+07$ & \\
\hline 6159.378 & 4.607 & -1.85 & 2.00 & $1.92 \mathrm{e}+08$ & \\
\hline 6165.360 & 4.143 & -1.524 & 2.00 & $8.77 \mathrm{e}+07$ & \\
\hline 6173.336 & 2.223 & -2.910 & 2.00 & $1.67 \mathrm{e}+08$ & \\
\hline
\end{tabular}


Table A1. continued

\begin{tabular}{|c|c|c|c|c|c|}
\hline $\begin{array}{c}\lambda \\
{[\AA]}\end{array}$ & $\begin{array}{r}\chi_{l} \\
{[\mathrm{eV}]}\end{array}$ & $\overline{\log g f}$ & $\delta \Gamma_{6}$ & $\begin{array}{l}\Gamma_{\mathrm{rad}} \\
{\left[\mathrm{s}^{-1}\right]}\end{array}$ & Note \\
\hline 6187.990 & 3.943 & -1.65 & 2.00 & $4.60 \mathrm{e}+07$ & \\
\hline 6200.313 & 2.608 & -2.48 & 2.00 & $1.03 \mathrm{e}+08$ & \\
\hline 6226.736 & 3.883 & -2.09 & 2.00 & $5.42 \mathrm{e}+07$ & \\
\hline 6229.228 & 2.845 & -2.885 & 2.00 & $1.45 \mathrm{e}+08$ & \\
\hline 6380.743 & 4.186 & -1.366 & 2.00 & $7.34 \mathrm{e}+07$ & \\
\hline 6385.718 & 4.733 & -1.82 & 2.00 & $2.34 \mathrm{e}+08$ & \\
\hline 6419.950 & 4.733 & -0.27 & 2.00 & $2.31 \mathrm{e}+08$ & \\
\hline 6591.313 & 4.593 & -1.99 & 2.00 & $1.40 \mathrm{e}+08$ & \\
\hline 6608.026 & 2.279 & -3.94 & 2.00 & $1.66 \mathrm{e}+08$ & \\
\hline 6653.853 & 4.154 & -2.38 & 2.00 & $2.09 \mathrm{e}+08$ & \\
\hline 6703.567 & 2.758 & -3.03 & 2.00 & $1.03 \mathrm{e}+08$ & \\
\hline 6710.319 & 1.485 & -4.79 & 2.00 & $1.66 \mathrm{e}+07$ & \\
\hline 6726.661 & 4.607 & -1.079 & 2.00 & $2.29 \mathrm{e}+08$ & \\
\hline 6733.151 & 4.638 & -1.45 & 2.00 & $2.27 \mathrm{e}+08$ & \\
\hline 6745.101 & 4.580 & -2.17 & 2.00 & $4.78 \mathrm{e}+07$ & \\
\hline 6745.957 & 4.076 & -2.74 & 2.00 & $3.79 \mathrm{e}+07$ & \\
\hline 6750.153 & 2.424 & -2.641 & 2.00 & $7.69 \mathrm{e}+06$ & \\
\hline 6806.845 & 2.727 & -3.11 & 2.00 & $1.02 \mathrm{e}+08$ & \\
\hline 6810.263 & 4.607 & -1.026 & 2.00 & $2.30 \mathrm{e}+08$ & \\
\hline 6820.372 & 4.638 & -1.16 & 2.00 & $2.22 \mathrm{e}+08$ & \\
\hline 7401.685 & 4.186 & -1.609 & 2.00 & $7.03 \mathrm{e}+07$ & \\
\hline 7418.667 & 4.143 & -1.476 & 2.00 & $1.06 \mathrm{e}+08$ & \\
\hline 7421.559 & 4.638 & -1.73 & 2.00 & $2.50 \mathrm{e}+08$ & \\
\hline 7440.952 & 4.913 & -0.662 & 2.00 & $4.97 \mathrm{e}+08$ & \\
\hline 7443.022 & 4.186 & -1.66 & 2.00 & $3.48 \mathrm{e}+07$ & \\
\hline 7453.998 & 4.186 & -2.36 & 2.00 & $1.45 \mathrm{e}+08$ & \\
\hline 7498.530 & 4.143 & -2.13 & 2.00 & $1.16 \mathrm{e}+08$ & \\
\hline 7507.261 & 4.415 & -0.972 & 2.00 & $9.77 \mathrm{e}+07$ & \\
\hline 7540.430 & 2.727 & -3.77 & 2.00 & $9.31 \mathrm{e}+07$ & \\
\hline 7547.910 & 5.100 & -1.247 & 2.00 & $6.38 \mathrm{e}+08$ & \\
\hline 7551.109 & 5.085 & -1.57 & 2.00 & $6.34 \mathrm{e}+08$ & \\
\hline 7582.122 & 4.955 & -1.64 & 2.00 & $1.00 \mathrm{e}+08$ & \\
\hline 7588.305 & 5.033 & -1.07 & 2.00 & $6.35 \mathrm{e}+08$ & \\
\hline 7745.500 & 5.086 & -1.149 & 2.00 & $6.37 \mathrm{e}+08$ & \\
\hline 7746.587 & 5.064 & -1.276 & 2.00 & $6.31 \mathrm{e}+08$ & \\
\hline 7751.137 & 4.991 & -0.775 & 2.00 & $6.44 \mathrm{e}+08$ & \\
\hline 7844.559 & 4.835 & -1.70 & 2.00 & $2.34 \mathrm{e}+08$ & \\
\hline \multicolumn{6}{|c|}{ Fe II; $\log \epsilon_{\odot}=7.51$} \\
\hline 5823.155 & 5.569 & -2.88 & 2.00 & $3.00 \mathrm{e}+08$ & not used \\
\hline 6149.258 & 3.889 & -2.84 & 2.00 & $3.39 \mathrm{e}+09$ & \\
\hline 6247.557 & 3.892 & -2.39 & 2.00 & $3.38 \mathrm{e}+08$ & \\
\hline 6369.462 & 2.891 & -4.20 & 2.00 & $2.90 \mathrm{e}+08$ & \\
\hline 6383.722 & 5.553 & -2.10 & 2.00 & $4.09 \mathrm{e}+08$ & \\
\hline 6416.919 & 3.892 & -2.69 & 2.00 & $3.37 \mathrm{e}+08$ & not used \\
\hline 6432.680 & 2.891 & -3.64 & 2.00 & $2.90 \mathrm{e}+07$ & \\
\hline 6446.410 & 6.223 & -1.88 & 2.00 & $5.22 \mathrm{e}+08$ & \\
\hline 6456.383 & 3.903 & -2.24 & 2.00 & $3.37 \mathrm{e}+08$ & \\
\hline 7449.335 & 3.889 & -3.168 & 2.00 & $4.09 \mathrm{e}+08$ & not used \\
\hline 7515.831 & 3.903 & -3.462 & 2.00 & $4.09 \mathrm{e}+08$ & \\
\hline
\end{tabular}

Table A1. continued

\begin{tabular}{|c|c|c|c|c|c|}
\hline $\begin{array}{c}\lambda \\
{[\AA]}\end{array}$ & $\begin{array}{r}\chi_{l} \\
{[\mathrm{eV}]}\end{array}$ & $\log g f$ & $\delta \Gamma_{6}$ & $\begin{array}{c}\Gamma_{\mathrm{rad}} \\
{\left[\mathrm{s}^{-1}\right]}\end{array}$ & Note \\
\hline \multicolumn{6}{|c|}{ Co I; $\log \epsilon_{\odot}=4.92$} \\
\hline 5647.234 & 2.280 & -1.58 & 2.50 & $1.66 \mathrm{e}+08$ & \\
\hline 6086.658 & 3.409 & 0.22 & 2.50 & $8.87 \mathrm{e}+07$ & \\
\hline 6093.143 & 1.740 & -2.37 & 2.50 & $2.08 \mathrm{e}+07$ & \\
\hline 6188.996 & 1.710 & -2.33 & 2.50 & $2.22 \mathrm{e}+07$ & \\
\hline 6257.572 & 3.713 & -1.08 & 2.50 & $6.89 \mathrm{e}+07$ & \\
\hline 6429.906 & 2.137 & -2.39 & 2.50 & $9.29 \mathrm{e}+05$ & \\
\hline 6632.433 & 2.280 & -1.87 & 2.50 & $6.46 \mathrm{e}+06$ & \\
\hline 6814.942 & 1.956 & -1.77 & 2.50 & $2.08 \mathrm{e}+07$ & \\
\hline 7417.367 & 2.042 & -1.97 & 2.50 & $2.22 \mathrm{e}+07$ & \\
\hline \multicolumn{6}{|c|}{$\mathbf{N i}$ I $\log \epsilon_{\odot}=6.25$} \\
\hline 5494.876 & 4.105 & -1.08 & 2.50 & $2.05 \mathrm{e}+08$ & \\
\hline 5578.711 & 1.676 & -2.68 & 2.50 & $5.43 \mathrm{e}+07$ & \\
\hline 5628.335 & 4.089 & -1.35 & 2.50 & $2.52 \mathrm{e}+08$ & \\
\hline 5638.745 & 3.898 & -1.72 & 2.50 & $1.27 \mathrm{e}+08$ & \\
\hline 5643.072 & 4.165 & -1.22 & 2.50 & $9.14 \mathrm{e}+07$ & \\
\hline 6025.751 & 4.236 & -1.69 & 2.50 & $1.92 \mathrm{e}+08$ & \\
\hline 6039.296 & 4.236 & -2.07 & 2.50 & $2.16 \mathrm{e}+08$ & \\
\hline 6086.276 & 4.266 & -0.51 & 2.50 & $2.54 \mathrm{e}+08$ & \\
\hline 6108.107 & 1.676 & -2.56 & 2.50 & $4.86 \mathrm{e}+07$ & \\
\hline 6111.066 & 4.088 & -0.86 & 2.50 & $1.46 \mathrm{e}+08$ & \\
\hline 6128.963 & 1.676 & -3.37 & 2.50 & $1.21 \mathrm{e}+07$ & \\
\hline 6130.130 & 4.266 & -0.963 & 2.50 & $2.78 \mathrm{e}+08$ & \\
\hline 6133.963 & 4.088 & -1.79 & 2.50 & $1.45 \mathrm{e}+08$ & \\
\hline 6176.807 & 4.088 & -0.27 & 2.50 & $1.45 \mathrm{e}+08$ & \\
\hline 6177.236 & 1.826 & -3.562 & 2.50 & $4.31 \mathrm{e}+07$ & \\
\hline 6186.709 & 4.105 & -0.922 & 2.50 & $2.06 \mathrm{e}+09$ & \\
\hline 6204.600 & 4.088 & -1.146 & 2.50 & $1.75 \mathrm{e}+08$ & \\
\hline 6370.341 & 3.542 & -1.957 & 2.50 & $1.48 \mathrm{e}+08$ & \\
\hline 6378.247 & 4.154 & -0.84 & 2.50 & $2.08 \mathrm{e}+08$ & \\
\hline 6424.847 & 4.167 & -1.354 & 2.50 & $2.13 \mathrm{e}+08$ & \\
\hline 6586.308 & 1.951 & -2.748 & 2.50 & $4.30 \mathrm{e}+07$ & \\
\hline 6598.593 & 4.236 & -0.94 & 2.50 & $1.92 \mathrm{e}+08$ & \\
\hline 6643.629 & 1.676 & -2.08 & 2.50 & $1.01 \mathrm{e}+08$ & \\
\hline 6767.768 & 1.826 & -2.24 & 2.50 & $1.03 \mathrm{e}+08$ & \\
\hline 6772.313 & 3.658 & -1.082 & 2.50 & $1.50 \mathrm{e}+08$ & \\
\hline 7414.500 & 1.986 & -2.22 & 2.50 & $1.03 \mathrm{e}+08$ & \\
\hline 7522.758 & 3.658 & -0.535 & 2.50 & $1.50 \mathrm{e}+08$ & \\
\hline 7525.111 & 3.635 & -0.64 & 2.50 & $1.19 \mathrm{e}+08$ & \\
\hline 7574.043 & 3.833 & -0.533 & 2.50 & $9.12 \mathrm{e}+07$ & \\
\hline 7826.766 & 3.699 & -1.856 & 2.50 & $5.98 \mathrm{e}+07$ & \\
\hline
\end{tabular}

